\section{Parenteral nutrition}

Deb Ghosh, specialist registrar in gastroenterology; Penny Neild, consultant gastroenterologist and honorary senior lecturer

Department of Gastroenterology,

St George's Hospital, London

The concept of parenteral nutrition (PN) was first explored in the 17th century when Christopher Wren infused wine, ale and opiates into the veins of dogs and reported his findings, ${ }^{1}$ but it was not until the 1960s that it became increasingly recognised as a medical therapy. ${ }^{2}$ With further advances in knowledge of PN formulation, aseptic techniques and so on, it has become a realistic feeding option for patients who have no other nutritional means available to them.

$\mathrm{PN}$ can be defined as the intravenous (iv) delivery of an artificial, nutritionally balanced combination of sterile nutrients. It is considered when all or part of nutrition cannot be provided via the enteral route. It may be beneficial in patients who are malnourished or at risk of malnutrition $^{3}$ and meet either of the following criteria:

- inadequate or unsafe enteral nutritional intake, or

- a non-functional, inaccessible or perforated (leaking) gastrointestinal tract (Table 1).

However, when used inappropriately or without due care, PN may be associated with significant and potentially life-threatening complications, as highlighted in the recent National Confidential Enquiry into Patient Outcome and Death report. ${ }^{6}$

This article discusses the indications, initiation, monitoring and stopping of $\mathrm{PN}$, the potential complications and how to minimise or avoid them.

\section{Indications for and decisions about parenteral nutrition}

When considering PN a number of questions must first be answered.

\section{Is it appropriate for this patient at this time?}

This initial question is one of the most important and often difficult decisions regarding PN. Further questions may be asked, which may help to clarify the position.

\section{Am I satisfied that enteral nutrition is unsafe, insufficient or has failed in this patient?}

Clear indications for PN include:

- extensive small bowel resection and residual short gut inadequate to maintain nutritional status, despite having tried all other measures

- peritonitis from a persisting small intestinal leak

- a newly developed proximal highoutput fistula.

In some patients, however, the decision is far less clear-cut, including:

- postoperative ileus

- severe acute pancreatitis

- distal small bowel fistula.

In the latter group, a time-limited trial of enteral (oral, nasogastric or postpyloric) nutrition, with or without prokinetics, may be attempted first.

Indications of lack of sufficient enteral absorption include:

- increased gastric residual volume (more than $250 \mathrm{ml} / 4 \mathrm{hr}$ )

- increasing abdominal distension

- persistent nausea/vomiting.
Absent bowel sounds in themselves do not signify lack of small bowel absorption.

\section{Has a clear end-point been agreed?}

When embarking on a course of PN it is vital to be clear about the objectives of the therapy. These may include:

- awaiting resolution of ileus

- being a 'bridge' to surgery in a patient with intestinal obstruction

- a time-defined trial of total PN in an effort to heal a new small intestinal fistula

- resolution of a small-bowel leak post-surgery

- a plan for home PN (HPN) in a patient with a short bowel or inoperable bowel obstruction.

In all cases, having such an objective specified will help to define the investigations, monitoring and planning and, where appropriate, guide the re-instigation of enteral nutrition.

\section{Initiation}

Both initiation and monitoring of $\mathrm{PN}$ in the hospital should ideally be undertaken by a multidisciplinary nutrition team, consisting of at least a dietitian, doctor, nutrition nurse and pharmacist. Such multiprofessional input will help to minimise the risk of potentially life-threatening mechanical, infective and metabolic complications.

Table 1. Intestinal failure. Intestinal failure 'results from obstruction, dysmotility, surgical resection, congenital defect or disease-associated loss of absorption and is characterised by the inability to maintain protein-energy, fluid, electrolyte or micronutrient balance'. ${ }^{4}$ Table reproduced with permission from Elsevier. ${ }^{5}$

Classification of intestinal failure

Type I - Short-term and reversible (eg postoperative/paralytic ileus, severe acute pancreatitis)

Type II - Prolonged but potentially reversible, associated with sepsis and metabolic complications

- Often related to abdominal surgery with complications or multiorgan failure

Type III - Long term and usually irreversible but stable

- Home parenteral nutrition often indicated

- Examples include short bowel syndrome, chronic malabsorption due to radiation enteritis and inflammatory bowel disease 


\section{Routes of administration}

PN may be administered either peripherally or centrally, depending on indication, duration of treatment, etc. Regardless of the route chosen, all lines should be inserted by skilled personnel, using strict aseptic technique and with a lumen dedicated exclusively for the use of PN.

\section{Central}

Central venous catheters range from multilumen subclavian/internal jugular lines to long-term venous access devices, such as tunnelled central catheters (eg Hickman) or totally implanted ports, depending on indication and available expertise. Peripherally inserted central catheters may also be used for up to three to six months, both in hospitalised patients and for HPN.

\section{Peripheral}

Peripheral PN may rarely be considered in patients requiring short-term $\mathrm{PN}$ $(<2$ weeks), but frequent catheter changes are necessary. Also, only PN solution with a low osmolarity (ideally $<800$ mosmol/l) should be administered via this route, to reduce the risk of thrombophlebitis or local tissue necrosis in the event of an inadvertently displaced cannula. Hence, it is not suitable for patients with poor peripheral access, higher nutritional requirements or those with strict fluid restrictions.

\section{Composition and administration of parenteral nutrition}

The quantity and proportion of macronutrients required for $\mathrm{PN}$ vary from patient to patient according to weight, illness and risk of refeeding syndrome (RS). Baseline biochemical assessment of micronutrients, electrolytes, renal and liver function, as well as fluid status, are also important to inform the composition of the PN.

Depending on the patient's calorie/nitrogen needs, $\mathrm{PN}$ will be provided either from an 'off-the-shelf' bag (pre-prepared, containing a specified quantity and proportion of protein, carbohydrate and fat, though usually no micronutrients) or a tailored bag, made from 'scratch' according to individual patient requirements. Electrolytes, trace elements and vitamins may either be administered simultaneously or added to the parenteral solution in the aseptic unit in the pharmacy.

$\mathrm{PN}$ is normally started as a continuous infusion over 24 hours. Once a patient is

\section{Key points}

Parenteral nutrition (PN) is a potentially life-saving form of nutrition support, but should be considered only for patients in whom enteral nutrition is unsafe, insufficient or has failed

Both initiation and monitoring of PN should be undertaken by a multidisciplinary nutrition team consisting of dietitian, nutrition nurse, nutrition pharmacist and gastroenterologist or other interested, suitably experienced clinician, to minimise the risk of potentially life-threatening mechanical, infective and metabolic complications

PN can be given short or long term; appropriate dedicated vascular access needs to be chosen accordingly

Choice of the composition and quantity of the PN bag needs to be individualised depending on baseline nutritional assessment, biochemical/electrolyte status and risk of refeeding syndrome (RS)

Catheter-related bloodstream infection and metabolic complications, particularly RS, are the most common serious complications of PN

KEY WORDS: catheter-related bloodstream infection (CRBSI), intestinal failure, nutrition support team, parenteral nutrition, re-feeding syndrome stable, this may change to cyclical delivery (over 12-16 hr). Such shorter infusion times allow the patient some freedom from being attached to their pump, but it is important to monitor blood glucose levels initially as some patients may experience rebound hypoglycaemia when the infusion is stopped.

\section{Monitoring}

In addition to clinical monitoring, including regular checks of the access site and anthropometric measurements, patients will require regular blood tests, particularly at initiation of $\mathrm{PN}$, to assess for significant electrolyte shifts, hyperglycaemia, and so on (Table 2). This is particularly important for patients who may be at risk of RS (see below). Trace elements ( $\mathrm{Zn}, \mathrm{Cu}, \mathrm{Se}$ ) and vitamin levels (B12, folate, D) should be measured on commencing PN. Patients on long-term PN should have three- to six- monthly checks of vitamins and trace element status.

\section{Complications}

The main complications of $\mathrm{PN}$ are mechanical, infective, or metabolic.

\section{Insertion/mechanical}

Careful line insertion by trained operators under aseptic conditions, preferably with the use of ultrasound (US) guidance, minimises mechanical complications (eg pneumothorax, blockage/kinking, line displacement).

Catheter-related thrombosis is best prevented by US-guided placement of lowest calibre catheter with good tip position. Treatment of thrombosis is by anticoagulation unless the catheter is infected or obstructed.

\section{Infective}

Catheter-related bloodstream infection $(\mathrm{CRBSI})^{7}$ is potentially life-threatening. It may occur either at the time of insertion if full aseptic technique is not followed, or subsequently as a 
consequence of suboptimal line care. The risk of CRBSI can be significantly reduced by scrupulous nursing care of the line, ensuring a dedicated lumen for provision of $\mathrm{PN}$ and careful handling of the PN bags. ${ }^{8}$ CRBSI is defined as ${ }^{7}$ :

- bacteraemia in a patient with an intravascular catheter and one or more positive blood cultures from a peripheral vein, clinical signs of infection and no apparent source for bloodstream infection with the exception of the catheter

- when quantitative or semiquantitative culture of the catheter isolates the same organism from the catheter segment and a peripheral blood sample.

On suspicion or diagnosis of CRBSI, indications for immediate line removal are as follows:

- tunnel infection or port abscess

- sepsis associated with haemodynamic compromise (shock)

- paired blood cultures positive for fungi or highly virulent bacteria

- complicated infection (eg evidence of endocarditis, septic thrombosis or other metastatic infections).

Otherwise, line salvage may be attempted in patients with 'precious' or long-term specially placed lines using the antibiotic lock technique. ${ }^{7}$

Empirical antibiotics for suspected CRBSI. Vancomycin is usually recommended because of its activity against coagulasenegative staphylococci and Staphylococcus aureus (including methicillin-resistant S. aureus). Additional empirical coverage for Gram-negative bacilli and pseudo- monas (ceftazidime etc) is recommended for severely ill or immunocompromised patients. Use of amphotericin B or iv fluconazole should also be considered for empirical treatment when fungaemia is suspected.

\section{Metabolic}

Hyperglycaemia is common, particularly in PN in acutely ill patients, and should be treated with insulin infusion to maintain serum glucose within the normal range and reduce the risk of potential sepsis. ${ }^{9}$

Abnormal liver function tests often develop in patients receiving $\mathrm{PN}$ and are commonly related to the underlying disease, medication, sepsis, and so on, rather than to PN per se. Cyclical feeding, avoidance of overfeeding or changing the composition of the feed, introduction of some enteral feeding, where possible, are different options to consider. ${ }^{10}$

Refeeding syndrome. The significant shifts in fluids and electrolytes that may occur in malnourished patients receiving nutrition (whether enteral or parenteral) is defined as RS. ${ }^{11}$ Hypophosphataemia is the biochemical hallmark of RS, though hypomagnesaemia and hypokalaemia are commonly also present.

During undernutrition, the body downregulates membrane pumping to conserve energy. This causes leakage of intracellular $\mathrm{K}, \mathrm{Mg}, \mathrm{Ca}$ and $\mathrm{PO}_{4}$, with subsequent whole-body depletion. Refeeding leads to reversal of the above process and, together with insulin-driven movements of electrolytes into cells, can lead to precipitous falls in circulating levels of $\mathrm{K}, \mathrm{Mg}$, $\mathrm{Ca}$ and $\mathrm{PO} 4 .^{11}$

Table 2. Monitoring of patients on parenteral nutrition. ${ }^{3}$

\begin{tabular}{lll} 
& On initiation & When stable \\
\hline Review for evidence of infection & Daily & Daily \\
Blood glucose & $4-6$ hourly & Daily \\
Plasma Na, K, Mg and PO4 & Daily & Weekly \\
LFT and full blood count & Weekly & Weekly \\
Trace elements (Zn, Cu, Se) and & On commencement and & $4-12$ weekly \\
vitamin levels (B12, folate, D) & periodically thereafter if & \\
& on long-term TPN & \\
\hline LFT = liver function test; TPN = total parenteral nutrition. &
\end{tabular}

When RS is severe and unrecognised clinical consequences are cardiac failure and arrhythmias, convulsions and coma, respiratory failure and muscle weakness/pain.

Patients at high risk of developing refeeding problems include those with low body mass index, significant recent unintentional weight loss or little or no nutritional intake in recent days (Table 3). A history of alcohol abuse or drugs including insulin, chemotherapy, antacids or diuretics further increases the potential risk. $^{3}$

RS is often missed unless considered from the outset of nutrition administration. For those at risk, nutrition support should begin at a maximum of $10 \mathrm{kcal} / \mathrm{kg} /$ day $(\leqslant 30 \%$ of energy requirements) with close monitoring of serum electrolytes and clinical condition, increasing slowly to meet full nutritional requirements over three to five days.

\section{Weaning from parenteral nutrition}

Alternative methods of feeding should always be established before weaning from PN. It should never be stopped abruptly as rebound hypoglycaemia can occur. Most patients can be converted entirely to enteral or oral feeding when this route meets more than half their total nutrient requirements.

\section{Home parenteral feeding}

HPN should be considered in stable patients on $\mathrm{PN}$ in whom intestinal failure is anticipated to last for longer than three months. In the UK, the most frequent indication is short bowel syndrome related to Crohn's disease. In the USA and parts of Europe, AIDS and cancer are the common indications. Patients considered for HPN should be referred to specialised centres with experience and back-up facilities. ${ }^{12}$

\section{Conclusions}

$\mathrm{PN}$ is a potentially life-saving means of artificial nutrition support in patients for whom there are no enteral alternatives. As more has been learned in recent years 
Table 3. Refeeding syndrome: how to identify patients at high risk of developing refeeding problems. ${ }^{3}$

Number of risk factors

\begin{tabular}{|c|c|}
\hline \multicolumn{2}{|c|}{ Number of risk factors } \\
\hline One or more of the following: & Two or more of the following: \\
\hline - $\quad$ BMI less than $16 \mathrm{~kg} / \mathrm{m}^{2}$ & - $\quad$ BMI less than $18.5 \mathrm{~kg} / \mathrm{m}^{2}$ \\
\hline - Unintentional weight loss $>15 \%$ in the last $3-6$ months & - Unintentional weight loss $>10 \%$ in the last $3-6$ months \\
\hline - Little or no nutritional intake for more than 10 days & - Little or no nutritional intake for $>5$ days \\
\hline - Low levels of $\mathrm{K}, \mathrm{PO}_{4}$ or $\mathrm{Mg}$ prior to feeding & $\begin{array}{l}\text { - History of alcohol abuse or drugs, including insulin, chemotherapy, } \\
\text { antacids or diuretics }\end{array}$ \\
\hline
\end{tabular}

about the adverse consequences of overfeeding and line sepsis, and with the advent and growth of multiprofessional nutrition support teams, it has also become a much safer form of treatment. However, it remains an invasive and relatively hazardous form of nutrition support and can be associated with risks from line placement, line infections, thrombosis and metabolic disturbance. It is therefore of the utmost importance to make very careful decisions about to whom, when and how this form of nutrition support should be given. PN should be initiated and monitored carefully by a multidisciplinary nutrition team to minimise potentially life-threatening complications.

\section{References}

1 Berry FB, Parker HS. Sir Christopher Wren: compleat philosopher. JAMA

1962;181:779-82.
2 Dudrick SJ, Wilmore DW, Vars HM, Rhoads JE. Can intravenous feeding as the sole means of nutrition support growth in the child and restore weight loss in an adult? An affirmative answer. Ann Surg 1969;169:974-84.

3 National Institute for Health and Clinical Excellence. Guidelines for nutrition support in adults. Clinical Guideline 32. London: NICE, 2006.

4 O'Keefe SJ, Buchman AL, Fishbein TM et al. Short bowel syndrome and intestinal failure: consensus definitions and overview. Clin Gastroenterol Hepatol 2006;4:6-10.

5 Shaffer J. Intestinal failure: definition and service development. Clin Nutr 2002;21(Suppl 1):144-5.

6 National Confidential Enquiry into Patient Outcome and Death. a mixed bag: an enquiry into the care of hospital patients receiving parenteral nutrition. London: NCEPOD, 2010.

7 Mermel LA, Farr BM, Sheretz RJ et al; Infectious Diseases Society of America, American College of Critical Care Medicine, Society for Healthcare Epidemiology of America. Guidelines for the management of intravascular catheter-related infections. Clin Infect Dis 2001;32:1249-72.

8 Cano NJ, Aparicio G, Carrero B et al. ESPEN Guidelines for adult parenteral nutrition, 2009. Clin Nutr 2009;28:359-479.

9 Van den Berghe G, Wouters P, Weekers F et al. Intensive insulin therapy in the critically ill patients. $N$ Engl J Med 2001;345:1359-67.

10 Lloyd DA, Gabe SM. Managing liver dysfunction in parenteral nutrition. Proc Nutr Soc 2007;66:530-8.

11 Solomon SM, Kirby DF. The refeeding syndrome: a review. J Parenter Enteral Nutr 1990;14:90-7.

12 Strategic framework for intestinal failure and home parenteral nutrition services for adults in England, April 2008.

Address for correspondence: Dr P Neild, Department of Gastroenterology, St George's Hospital, Blackshaw Road, London SW17 OQT.

Email: penny.neild@nhs.net 